\title{
Un-crewed aerial vehicle population survey of three sympatrically breeding seabird species at Signy Island, South Orkney Islands
}

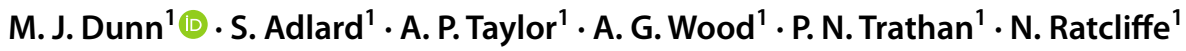

Received: 20 July 2020 / Revised: 4 February 2021 / Accepted: 10 February 2021 / Published online: 3 March 2021

(c) Crown 2021

\begin{abstract}
Surveying seabirds in polar latitudes can be challenging due to sparse human populations, lack of infrastructure and the risk of disturbance to wildlife or damage to habitats. Counting populations using un-crewed aerial vehicles (UAVs) is a promising approach to overcoming these difficulties. However, a careful validation of the approach is needed to ensure comparability with counts collected using conventional methods. Here, we report on surveys of three Antarctic bird species breeding on Signy Island, South Orkney Islands; Chinstrap (Pygoscelis antarctica) and Gentoo (Pygoscelis papua) Penguins, and the South Georgia Shag (Leucocarbo atriceps georgianus). We show that images from low-altitude UAV surveys have sufficient resolution to allow separation of Chinstrap Penguins from contiguously breeding Adélie Penguins (Pygoscelis adéliae), which are very similar in appearance when viewed from overhead. We compare data from ground counts with manual counts of nesting birds on images collected simultaneously by low-altitude aerial photography from multi-rotor UAVs at the same colonies. Results at this long-term monitoring site confirmed a continued population decline for Chinstrap Penguins and increasing Gentoo Penguin population. Although both methods provided breeding pair counts that were generally within 5\%, there were significant differences at some locations. We examine these differences in order to highlight potential biases or methodological constraints that should be considered when analysing similar aerial census surveys and comparing them with ground counts.
\end{abstract}

Keywords Un-crewed aerial system · UAV · Seabird · Survey · Population · South Orkney Islands · Ecological variability · Pygoscelis penguins

\section{Introduction}

Understanding patterns of abundance in wildlife populations-including those of seabirds—-through space and time, is dependent on the collection of accurate counts at appropriate frequency and scales (Lynch et al. 2015; Hodgson et al. 2018; Rush et al. 2018). In the case of seabirds, which are one of the most threatened of all bird groups globally (Croxall et al. 2012), such information can be critical to conservation efforts as it allows species at global risk to be recognised and the importance and condition of specific populations to be determined, including in the Antarctic (Harris et al. 2015;

P.N. Trathan and N. Ratcliffe have contributed equally to the study.

M. J. Dunn

mdunn@bas.ac.uk

1 British Antarctic Survey, Natural Environment Research Council, Madingley Road, Cambridge CB3 0ET, UK
Trathan et al. 2015; Phillips et al. 2016; Southwell et al. 2017a). However, obtaining such data from polar regions is inherently difficult owing to the lack of infrastructure and volunteer networks that are available at lower latitudes: conventional ground-based counts are only possible close to research stations or during short visits to particular locations on ships of opportunity (Naveen et al. 2000; Lynch et al. 2008). Fortunately, a number of developing technologies have recently become available to seabird researchers, all of them providing means of overcoming to some extent the challenges of accessing polar seabird populations. These include the use of remotely sensed satellite imagery to locate and estimate the size of colonially breeding bird populations (Fretwell et al. 2012; Lynch et al. 2012; Fretwell et al. 2015), and autonomous time-lapse camera systems collecting spatially extensive phenological and reproductive data, replacing the need for direct observation (Southwell et al. 2013; Lynch et al. 2015; Black et al. 2018; Hinke et al. 2018). However, whilst satellites can achieve global coverage, 
they also have relatively low accuracy (LaRue et al. 2014; Fretwell et al. 2017) and occasional errors in colony identification (Southwell et al. 2017b), whereas autonomous camera systems have too narrow a field of view to accurately monitor numbers except in small colonies, making them more suitable for study of nesting phenology and success than census work (Black et al. 2018; Hinke et al. 2018). Manned aerial seabird surveys have been successfully utilised in the polar regions (Trathan 2004) but are expensive and require either runways or ships with helidecks to operate.

The use of un-crewed aerial vehicles (UAVs) has, in recent years, offered an alternative tool for carrying out systematic census work on colonial, surface-nesting seabird species across a wide variety of environments (Sarda-Palomera et al. 2012; Barr et al. 2018; Rush et al. 2018), including within the Antarctic and islands of the South Atlantic (Ratcliffe et al. 2015; Pfeifer et al. 2019; Oosthuizen et al. 2020). Ground counts are a well-established means of accurately assessing numbers of breeding seabirds (CCAMLR 2004). However, following a number of concept studies at small scales, the increasing reliability, capability and affordability of different UAV platforms have led to a growing number of ambitious, large-scale seabird population surveys spread across archipelagos or large islands using vertically captured, high-resolution imagery (Goebel et al. 2015; Borowicz et al. 2018; Korczak-Abshire et al. 2019).

UAVs provide a means of quickly and accurately surveying seabirds across large areas at high spatial resolution that would be otherwise unobservable, such as offshore stacks, tree canopy or wetlands (Linchant et al. 2015; Hodgson and Koh 2016; Barr et al. 2018; Hodgson et al. 2018). In rugged and uneven terrain, aerial imagery from UAVs can reveal nests that might otherwise remain hidden from a groundbased perspective (Goebel et al. 2015; Brisson-Curadeau et al. 2017). Crucially, imagery from UAVs can be reanalysed at a later date, as the images provide a permanent record from each survey, and potentially a wealth of additional data on colony distribution, area, shape, nest spacing and nest/colony habitat selection (Henriksen et al. 2015; Chabot and Francis 2016; Rush et al. 2018).

UAVs potentially offer a means of reducing disturbance to breeding seabirds (or other non-target species) by eliminating the need for intrusion into colonies on foot (McClelland et al. 2016; Borrelle and Fletcher 2017; Brisson-Curadeau et al. 2017). Although the application of UAVs continues to increase, including in the polar regions, there is concern over potential flight disturbance impact on seabirds and other marine wildlife, with a number of studies seeking to establish safe operating procedures to prevent wildlife disturbance (Borrelle and Fletcher 2017; Brisson-Curadeau et al. 2017; Rummler et al. 2018; Weimerskirch et al. 2018). Based on data from existing studies using UAVs, current recommendations relating to the use of UAVs on Antarctic wildlife by the Scientific Committee on Antarctic Research (SCAR) highlight the need for further studies evaluating the use of such platforms in wildlife research (Mustafa et al. 2018). Nevertheless, given the known risks of ground survey disturbance to breeding colonial birds (Carney and Sydeman 1999), when used appropriately, UAVs are able to collect comparable data in a significantly shorter time than groundbased surveys of the same-sized colonies, without undue disturbance (Rush et al. 2018; Vas et al. 2018; Valle and Scarton 2020).

There are also some drawbacks to the use of UAVs, compared with ground surveys: reduced opportunities to carry out surveys owing to inclement weather preventing flights, potential for loss or failure of equipment curtailing surveys and potential difficulties in obtaining permits at both national and local levels (Ratcliffe et al. 2015; McClelland et al. 2016; Callaghan et al. 2018). A need for trained personnel and capital investment needed to procure equipment can also limit wider application, although the recent growth of the consumer-grade UAV market mean UAVs are now easier to fly and more affordable than was previously the case (Rummler et al. 2015; Hodgson et al. 2018; Mustafa et al. 2018).

Modern UAVs commonly used for wildlife surveys can be classified into two main categories: multi-rotor and fixedwing (Verfuss et al. 2019). Fixed-wing UAVs offer a longer flight time and greater survey range than multi-rotor UAVs but require a flat area for launch and landing (Verfuss et al. 2019). Their higher flight speeds, lower manoeuvrability and operation beyond visual line of sight require higher safe operational altitudes, resulting in a reduction in image resolution which impairs identification of similar species in mixed species colonies and separation between breeding and non-breeding birds (Zmarz et al. 2018; Korczak-Abshire et al. 2019; Pfeifer et al. 2019). Fixed-wing platforms are therefore most suited to surveys of single-species colonies, although differences in nest spacing patterns and habitat type may allow separation of species in some circumstances (Zmarz et al. 2018). Multi-rotor platforms are small, portable and have vertical take-off and landing capability allowing deployment from boats or rugged terrain (Goebel et al. 2015; Ratcliffe et al. 2015; Verfuss et al. 2019). Their slow flight speed and high manoeuvrability allow low-altitude surveys and hence higher-resolution images compared to fixed-wing UAVs and manned flights (Borowicz et al. 2018; Hodgson et al. 2016, 2018), making it possible to separate species in mixed colonies by their field characteristics and non-breeders by their posture (Rush et al. 2018; Magness et al. 2019). Both platform types therefore have complementary roles in surveying of seabird colonies, with fixed-wing UAVs being most suitable for long-range surveys of single species colonies from a flat launch site and multi-rotors for 
short-range surveys of mixed species colonies from boats or rough ground.

The Western Antarctic Peninsula and Scotia Sea have been identified as regions undergoing rapid environmental change, including increasing air and ocean temperatures and reductions in seasonal sea-ice extent and duration (Vaughan et. 2003; Forcada et al. 2006; Ducklow et al. 2007; Stammerjohn et al. 2008a, b; SCAR 2009; Turner et al. 2016). Across the region, seabird census data (in particular for three species of Pygoscelis penguins which are similar in appearance and breed sympatrically at numerous sites) have revealed significant population trends that have in turn been linked to changes in their environment, especially sea ice (Fraser et al. 1992; Trathan et al. 1996; Lynch et al. 2012), and regional environmental indices such as the Southern Annual Mode (Forcada and Trathan 2009) and the El Niño-Southern Oscillation (Trathan et al. 2006, 2007; Trivelpiece et al. 2011). Seabirds thus represent a practical indicator of change in the Antarctic marine ecosystem. Improving accuracy, frequency and spatial extent of counts is important to not only understand the dynamics driving specific populations, but to provide the latest site-wide census data needed to correct bias in size distribution of local-scale counts and in so doing contribute to seabird abundance monitoring at a regional scale (Lynch et al. 2012, 2013; Dunn et al. 2016).

Here we describe an island-wide population survey using multi-rotor UAVs to collect aerial images of three colonial, surface-nesting seabird species breeding at Signy Island, South Orkney Islands, during the austral summers 2016/2017 and 2017/2018. We selected multi-rotor UAVs as the survey required high-quality images to identify incubating Chinstrap Penguins (Pygoscelis antarctica), Gentoo Penguins (Pygoscelis papua) and South Georgia Shags (Leucocarbo atriceps georgianus) and separate these from nonbreeding conspecifics and sympatrically breeding Adélie Penguins (Pygoscelis Adéliae) that were not included in the census as the timing of the survey was too late in their nesting season. We compared count data collected using UAV aerial imagery with simultaneous ground counts of the same colonies to determine comparability between UAV and ground counts and to allow for a sound quantitative adjustment for any method-related differences in data bias between protocols. We also tested the potential for UAVs to extend counts to nearby islets that were previously inaccessible owing to a lack of boat transportation. Additionally, we present the latest (2017/18) whole-island counts of Chinstrap and Gentoo Penguins at Signy Island and compare these with previous surveys.

\section{Materials and methods}

\section{Study site and species}

Our study was carried out at Signy Island, South Orkney Islands $\left(60^{\circ} 42^{\prime} \mathrm{S}, 45^{\circ} 36^{\prime} \mathrm{W}\right.$, Fig. 1). The British Antarctic Survey established a research station on the island in 1947 and has since undertaken long-term research on penguins and other taxa, so the distribution and population trends are well known for a number of seabird species (Dunn et al. 2015, 2016). Gentoo Penguins breed at the northern end of the island (North Point), whilst Chinstrap Penguin colonies are located at North Point, Gourlay Peninsula, the southwest coast (Fyr Channel) and on several offshore islands (Confusion, Moe, Mariholm and Oliphant, Fig. 1), although access to the offshore islands is limited owing to boats being unavailable. A census in 2009/2010 counted 1,300 pairs of Gentoo Penguins and 19,500 Chinstrap pairs breeding at Signy (Dunn et al. 2016). Although not part of this study, there are also approximately 18,300 pairs of Adélie Penguins breeding at Signy (Dunn et al. 2016). All penguin species nest sympatrically at Signy: Chinstrap colonies occupy sloping ground with large boulders and rock outcrops, whilst Gentoo colonies are found on higher ground and elevated headlands usually $\sim 20 \mathrm{~m}$ from the nearest Chinstrap colony (White and Conroy 1975; Volkman and Trivelpiece 1981; Williams 1995). On Signy, the difference in breeding chronology between Adélie and Chinstrap Penguins results in the former beginning their breeding season two to three weeks earlier. Although this results in little intermixing of nest sites between the two species, it is not uncommon at Signy for nesting Chinstraps to settle around the edge of Adélie colonies, creating contiguous boundaries. Gentoo colonies are discrete from those of the other species. An estimated total island population of 306 breeding pairs of South Georgia Shags have been recorded during the 2016/2017 breeding season at Signy (Dunn 2020). South Georgia Shags breed on sea cliff ledges and slopes at two locations: North Point on the main island and Shagnasty Island (Fig. 1) that is accessible on foot during low spring tides.

\section{Field equipment used}

The UAV we used during the 2017/2018 breeding season was an Inspire 2 quadcopter (DJI, Shenzhen, China; https:// www.dji.com/uk/inspire-2) equipped with a DJI Zenmuse X5s Camera with Micro 4/3 Sensor and wide-angle fixed lens DJI MFT 15 mm/1.7 ASPH 24 mm equivalent (https:// www.dji.com/zenmuse-x5s/info). The Inspire 2 has approximately 20 minutes of flight time on a single charge. A second aircraft, the DJI Phantom 4 Pro quadcopter with integrated 

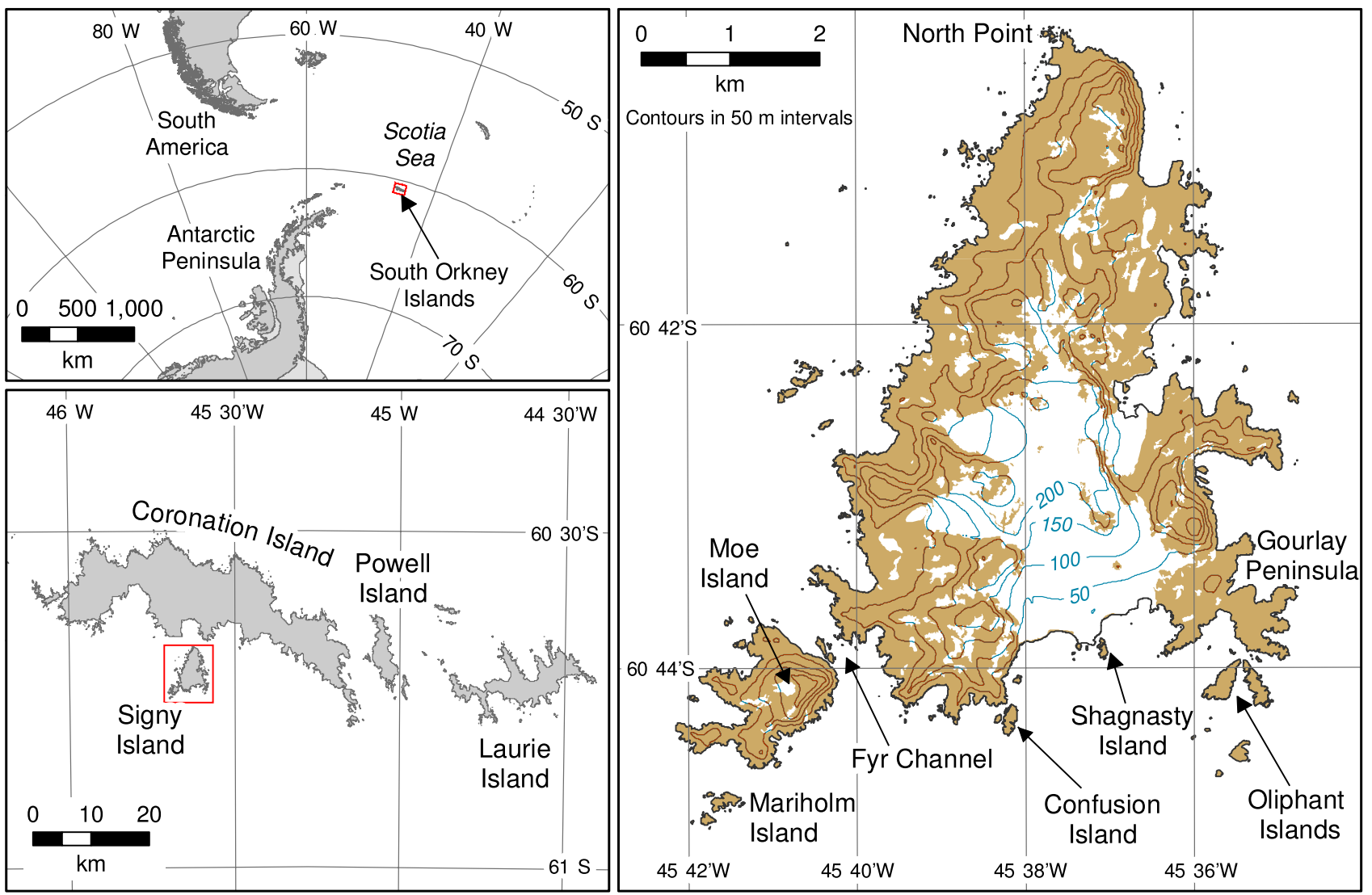

Fig. 1 Location of South Orkney Islands: locations of Chinstrap and Gentoo Penguin and South Georgia Shag breeding colonies on Signy Island are shown—see text for further details

camera mounting a FOV $94^{\circ} 20 \mathrm{~mm}$ f/2.8 lens and similar flight duration, was used for the 2016/2017 season and partly in the 2017/2018 season (https://www.dji.com/uk/phantom4-pro). It is smaller and less robust than the Inspire 2, but more suitable for hand launches and captures on uneven terrain. In the case of both UAVs, a sufficient number of batteries were taken into the field each day to complete the planned surveys. Batteries were subsequently recharged at the research station.

\section{Survey methods}

Surveys of the same colonies were conducted using counts by observers on the ground and aerial surveys using UAVs: ground counts took place immediately following the UAV survey at each colony. Ten Gentoo and eleven Chinstrap colonies were surveyed during 2016/2017 using both direct counts and UAVs. In 2017/2018, an all-island survey of Chinstrap Penguins (104 colonies in total) was carried out by UAV (including all of Signy and the offshore islands Confusion, Oliphant and Shagnasty), with ground counts also conducted for 56 of the mainland colonies. The offshore island counts were omitted in previous years as they were not accessible. Both of the South Georgia shag colony areas on Signy were surveyed during 2017/2018 (ground counts and UAV aerial surveys).

Ground surveys of penguin colonies followed the methods detailed by the Commission for the Conservation of Marine Living resources (CCAMLR) Ecosystem Monitoring Programme (CEMP) (CCAMLR 2004). Each spatially discrete group of breeding birds was defined as a colony and complete counts made of each (CEMP Method A3A), (CCAMLR 2004). The count unit was apparently occupied nests (AONs), which gives an approximation of the number of breeding pairs. An AON was defined as a bird lying or sitting on a ring of pebbles. Surveys must be conducted within one week of the peak of egg laying to be accurate: too early and later laid AONs will be missed and too late and a proportion of AONs will have failed. Optimal dates for the Chinstrap surveys were therefore determined from study colonies where nesting phenology data were collected every three days (CCAMLR 2004). Chinstrap counts took place between 8 and 24th December in both 2016/2017 and 2017/2018, and Gentoo surveys took place on the 29th November in the 2016/2017 season only. The surveys were too late in the season to be accurate for the earlier nesting 
Adélie Penguins, but separating them from the very similar looking Chinstrap penguins in mixed colonies presented a key challenge for the UAV surveys (see below).

For South Georgia Shag surveys, optimal survey dates were based on observed phenology during approximately weekly visits to the North Point colony in each survey year. When the peak of laying was reached, all South Georgia Shag AONs (birds sitting on a pedestal nest) were counted. During the 2017/2018 season, laying peaked on 19th and 20th December, but the date varied between 13th November and 27th December between 1996/1997 to 2019/2020 (BAS unpublished data). Counts of the Shagnasty Island colony were also conducted in 11 of the intervening years.

\section{Ground count protocol}

Ground surveys were carried out using: (a) visual scan counts with a tally counter, conducted from the edge of the colony or adjacent vantage points of between 30-50 m distance using binoculars (repeated by two observers until counts were within $10 \%$ of one another); (b) taking an oblique digital photograph (Canon EOS 60D Digital SLR with Canon EF-S 17-55 mm f/2.8 IS USM lens) of the colony from a vantage point and then counting individual occupied nests on the images taken or; (c) using capture-mark counts involving observers walking through the colony and spraying each AON with wildlife-safe livestock marker dye to avoid omission or double counting of nests (CCAMLR 2004). Method (c) was only used for smaller colonies $(<400$ AONs), although in some cases counts at several adjacent sub-colonies were made using this method and combined. As Method (c) resulted in some disturbance to incubating birds (birds sometimes left their nests but usually returned quickly), such counts were only conducted after the UAV surveys had been completed.

\section{Un-crewed aerial vehicle survey protocol}

All flights took place in dry conditions in wind speeds below 15 knots $\left(7.7 \mathrm{~ms}^{-1}\right)$. Survey areas were marked out with coloured plastic sheets weighed down with stones; GPS coordinates of these were recorded to allow subsequent georeferencing of images. A cloth "helipad" was used to protect the drone from dust and guano during take-off and landing, which was positioned on a flat area at least $30 \mathrm{~m}$ distant from any nesting birds to reduce disturbance (Junda et al. 2015; Mustafa et al. 2018; Rummler et al. 2018). The location and name of the colony were written on a small whiteboard, and a photograph of this was taken with the UAV camera to allow subsequent image identification. Camera SD cards were changed after every flight to secure the data in case the drone was lost subsequently. An observer assisted the pilot with flight preparations, warned of potential hazards during flights (such as the approach of flying birds or squalls) and recorded behavioural responses of the birds to disturbance.

The UAVs were flown manually rather than on preplanned routes as most colonies were relatively small and often located in areas of complex terrain. Surveys were conducted at a constant altitude of $35 \mathrm{~m}$ above ground level, during which overlapping images were taken using a "lawnmower" flight pattern to cover the entire colony area (Fig. 2a). This altitude provided a good compromise between the swath of the transect, image resolution and risk of disturbance to birds (Mustafa et al. 2018). The resolution from this altitude was sufficient to discriminate between similar penguin species nesting in mixed colonies (see below). We found altitudes higher than $35 \mathrm{~m}$ above ground reduced the resolution of the images, making identification less certain. Most images were taken with a vertical camera angle but, where colonies occupied broken terrain, oblique images were also taken to detect birds that were not visible from above. The average time taken to survey each colony using a UAV was five minutes.

The shags nesting offshore are infrequently visited by researchers, leading to concern that these individuals might be particularly sensitive to disturbance. As a precaution, we increased the UAV survey height to $50 \mathrm{~m}$ (for both shag colonies to maintain consistency). Shags have large pedestal nests and are very different in appearance from contiguously nesting chinstrap penguins, so identification from this altitude did not present problems.

\section{Data analyses}

Images were processed and nests counted immediately after collection. Adobe Lightroom (https://www.adobe.com) was used to download, edit, batch-name and archive all images, and a panoramic stitch software, AutoPanoGiga Pro (http:// www.autopano.net), was used to create mosaic images for colonies surveyed with multiple frames. Adobe Photoshop (https://www.adobe.com) was used to label the stitched mosaics and zoom in to analyse image clarity and accuracy. Fiji-Image $\mathbf{J}$ (https://imagej.net/Fiji), an open-source free counting software, was used to manually count nests in the images. Each bird in an image was inspected and labelled according to species and whether or not it qualified as an AON. Where this was difficult to determine in aerial photographs, other clues such as bird body posture and guano streaks around the nest and on the back of individual birds were used to help differentiate between incubating and offduty/non-breeding birds (Fig. 3).

Careful attention was necessary to omit unoccupied nests and non-breeding birds, the latter distinguished as standing upright as opposed to lying down in an incubation posture, or lying/standing on ground with no signs of nesting material beneath them. Other species not targeted for the survey were 
a

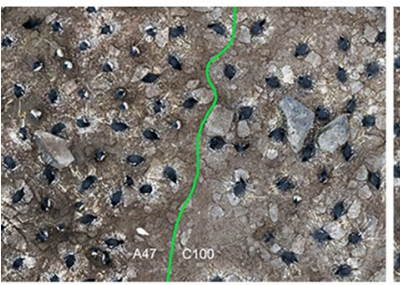

\section{Adelie}

C Pygoscelis adeliae
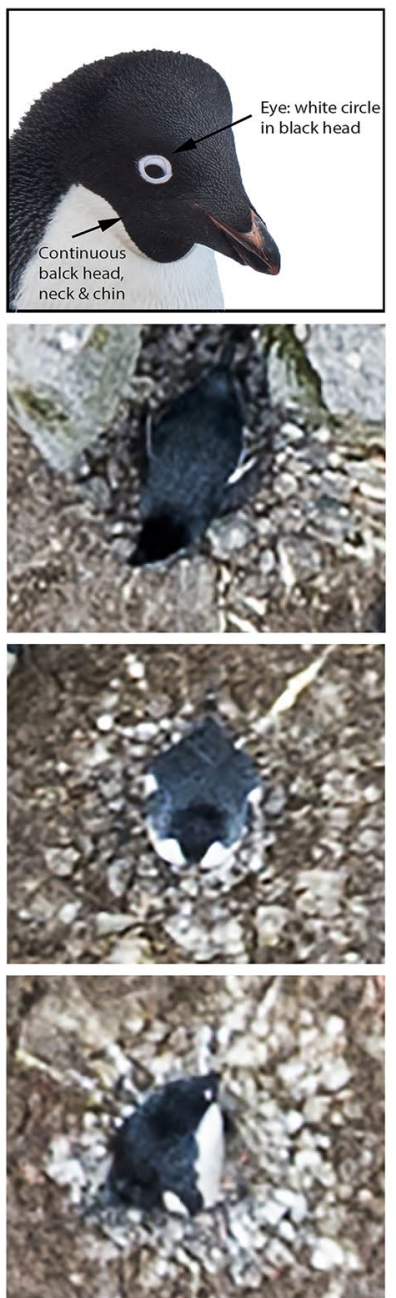

b

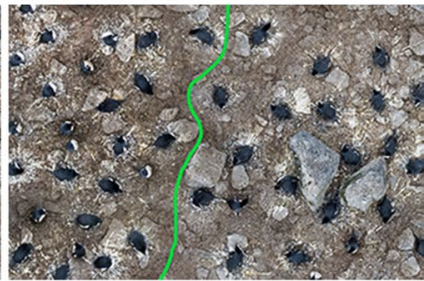

Chinstrap

Pygoscelis antarcticus
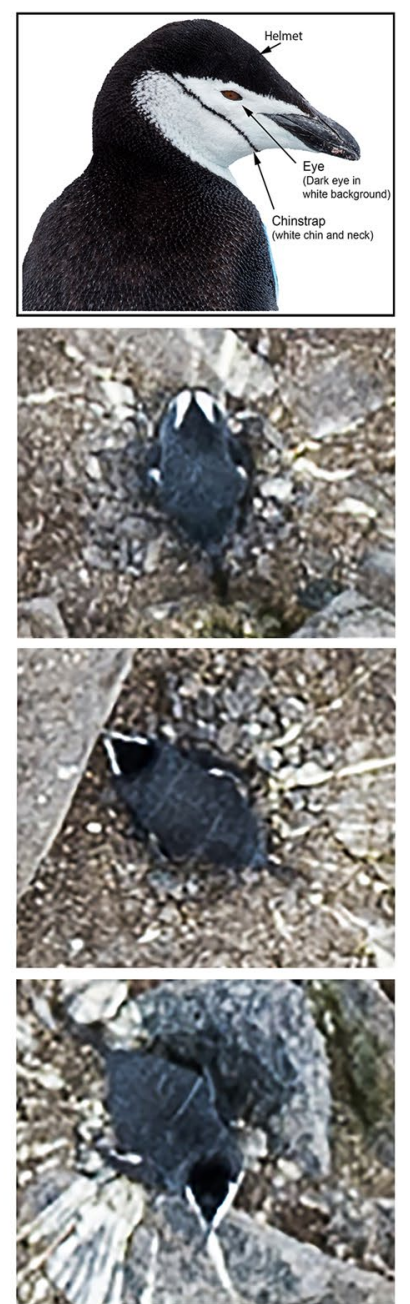

Fig. 2 UAV images of adjacent Chinstrap Penguin colony C100 (right of green line) and Adélie Penguin colony A47 (left of green line), Signy Island. $a=$ image taken at $35 \mathrm{~m}$ height. $\mathrm{b}=$ magnified image allowing most nesting birds to be identified to species. The green line represents the boundary between the Adélie and Chinstrap colonies. Adélies are to the left of the line and chinstraps to the right. $\mathrm{c}=$ Morphometric features used to distinguish between Adélie and Chinstrap Penguins

also omitted: in most cases, this was Adélie Penguins nesting immediately adjacent to chinstrap colonies. Species were distinguished based on their size and markings: Chinstrap Penguins were discriminated from Adélies by their white facial

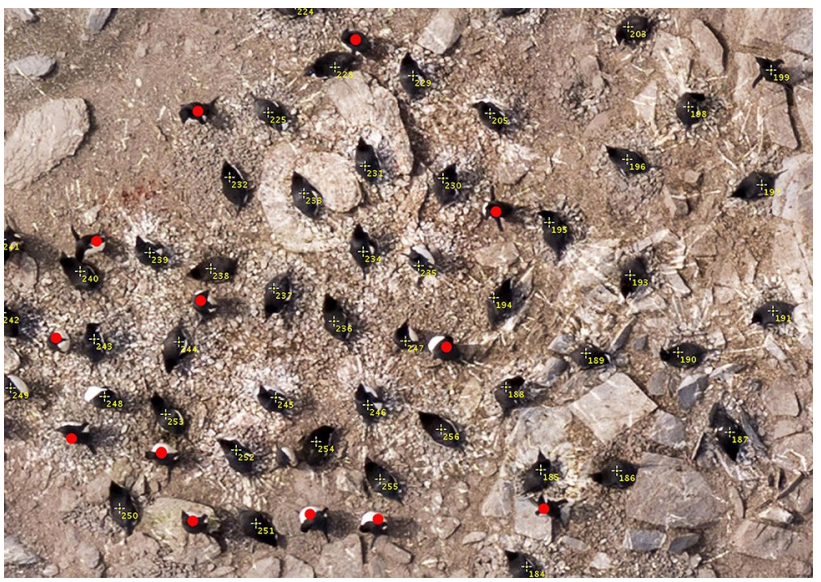

Fig. 3 Examples of non-breeding Chinstrap Penguins (red dots) and apparently occupied nests: distinguishing features include posture, presence/absence of nest material, presence/absence and radius of guano streaks and guano on back of bird

plumage and smaller area of black head colouration although this character is subtle when viewed from above and requires high-resolution images for it to be visible (Fig. 2b, c). Occasionally penguins were unidentifiable and were either discounted if located along a shared colony boundary, or else classed as the same species as neighbouring birds. Gentoo Penguins were easily distinguishable from both Adélie and Chinstrap Penguins by their white head markings, larger size and orange beak. The six Gentoo Penguin colonies were also located some distance away from Adélie and Chinstrap colonies.

Paired t-tests were used to compare whether counts made at the same colonies, within years and species, differed significantly. A standard linear regression model was used to examine the relationship between UAV and ground count data across all penguin species and years combined. We used the function linearHypothesis from the CAR package in Program R, to test whether the slope of each regression differed significantly from one (i.e. representing a 1:1 correspondence between UAV and ground count data). We used the Cook's D statistic from the influencePlot function within CAR to identify any outliers that had a large influence on the slope estimate. If these were evident, we fitted a robust regression model with Huber weights using the MASS package in program $\mathrm{R}$, which reduces the influence of outliers on the slope estimate.

\section{Results}

\section{Comparisons of ground and un-crewed aerial vehicle counts}

In 2016/2017, comparison of ground counts with UAV counts at a sample of 16 Chinstrap Penguin colonies on the 
same day estimated 1384 and 1352 AONs, respectively, a-2.3\% difference (Online Resource 1). In 2017/2018, all Chinstrap Penguin colonies and sub-colonies (104) were surveyed on Signy using UAVs and 56 of these were also surveyed using ground counts. UAV counts estimated 6006 and ground counts 6345 AONs, a difference of $-5.3 \%$ (Online Resource 2). Paired $t$-tests at the scale of individual colonies showed that these small differences were not significant in either year between the 11 Chinstrap Penguin colonies in 2016/2017 ( $t=-1.11$, degrees of freedom $(d f)=10, p=0.294)$ and 56 Chinstrap Penguin colonies in $2017 / 2018(t=1.36, d f=55, p=0.1799$, Fig. 4). All six Gentoo Penguin colonies on Signy were surveyed by both UAV and ground count methodology in 2016/2017. There was a $-0.2 \%$ difference between Gentoo Penguin pair numbers recorded from UAV aerial imagery (914 pairs) compared to ground counts (916 pairs, Online Resource 3). The six Gentoo Penguin colonies showed no significant difference between UAV imagery and ground surveys $(t=0.11, d f=5$, $p=0.9154$, Fig. 4). Pooling penguin species and years, a linear regression of UAV counts against ground surveys at the same colonies produced a positive relationship with a slope of 1.08 (standard error $(\mathrm{SE})=0.02, n=73$ ), which differed significantly from one $(F=17.36, d f=1, p<0.0001$, Fig. 4). The count of the largest Chinstrap colony was an obvious negative outlier that exerted a large leverage on the slope (Cook's $D=9.23$ ), where UAV counts estimated numbers $17.8 \%$ higher than the model estimate (Fig. 4, Online

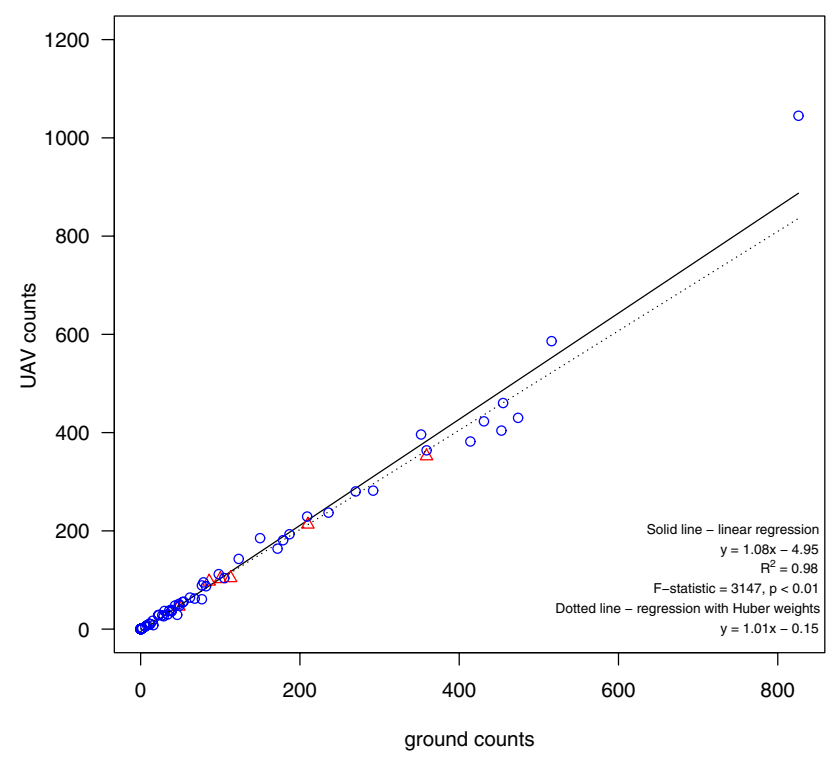

Fig. 4 Comparison of UAV and ground survey counts for penguin colonies, Signy Island: Linear regression lines are shown, with standard linear regression as solid line and regression including Huber weights shown as dashed line. Regression equations are shown in the plot. Blue circles indicate Chinstrap colonies (2016/2017 and 2017/2018) and red triangles indicate Gentoo colonies (2016/2017)
Resource 2). The robust regression with Huber weights reduced the regression slope to $1.01(\mathrm{SE}=0.003, n=73$, Fig. 4). Although this slope was very close to one, it still differed significantly from one $(F=13.49, d f=1, p<0.0001)$, owing to the very small standard error of the estimate.

A total of 304 South Georgia Shag AONs were identified from UAV images and 281 pairs were identified from ground counts in 2017/2018 (Online Resource 4), equating to a difference of $+8.2 \%$. However, ground counts and UAV surveys recorded 129 AONs and 130 AONs, respectively, at the Shagnasty Island colony, and 152 and 174 AONs at the North Point colony; a difference of $+0.8 \%$ and $+14.5 \%$, respectively, between ground and UAV counts.

Only minor behavioural responses were observed from any of the target species surveyed during UAV flights: no breeding birds were observed to leave their nests during UAV surveys, but during landings some head-turning was noted in Chinstrap Penguins at the border of colonies $30 \mathrm{~m}$ away. No collisions between the UAVs and flying seabirds occurred, even though skuas (Stercorarius spp.) and petrels (Southern Giant Petrel Macronectes giganteus, Cape Petrel Daption capense, snow petrel Pagodroma nivea and Wilson's Storm Petrel Oceanites oceanicus) utilised the airspace above the surveyed colonies.

\section{Penguin population counts and trends}

The total number of Chinstrap Penguin AONs counted by UAVs in 2017/2018 was 19,419 (Online Resource 5). The last all-island Chinstrap Penguin count in 2009/2010 did not include offshore islands, so excluding these produces a total of 18,137 AONs for the mainland of Signy alone in 2017/2018 (Online Resource 5). This equates to a decrease of $7.1 \%$ ( $-0.9 \%$ per annum) from the previous Chinstrap Penguin survey (ground count) of 19,530 pairs carried out on Signy in 2009/2010 (Dunn et al. 2016).

All six Gentoo Penguin colonies on Signy were surveyed by both UAV and ground count methodology in 2016/2017 (Online Resource 3). Overall, AON numbers from each count method both showed the same increase in Gentoo pairs of $21.4 \%$ (equalling $+2.8 \%$ per annum) since the 2009/2010 Gentoo Penguin ground count (Dunn et al. 2016).

\section{Discussion}

This study shows close comparability between ground counts and UAV surveys for three different species of Antarctic seabird. A 5\% threshold for count repeatability has been recommended in previous studies reporting the size of penguin populations in the Antarctic (Woehler and Croxall 1997; Naveen et al. 2000; CCAMLR 2004; Borowicz et al. 2018) and in our survey counts of penguin colonies using 
UAVs and ground counts within colonies on the same day were at or below this value. The regression of UAV counts against ground counts showed a slope value of 1.01, and while this differed significantly from a 1:1 correspondence, it falls within an accuracy threshold of $\pm 5 \%$. This conforms with our practical requirements for estimates to be within 5\% of each other to be considered comparable. Previous studies have also found that UAV counts are similar to ground counts (Chabot et al. 2015; Hodgson et al. 2016, 2018), thus allowing direct comparisons of trends through time or relative abundance through space across methodologies without the need for correction factors.

The largest Chinstrap Penguin colony showed the highest deviation from a 1:1 count ratio, with larger numbers of nests counted from UAV images than by ground count estimates. The ground counts for this colony (C65) were carried out from adjacent vantage points at a distance of between $30-50 \mathrm{~m}$ using binoculars and a tally counter. We believe the higher number of AONs recorded from UAV images reflects visual count underestimates, as has been reported in previous studies (Frederick et al. 2003; Buckland et al. 2012; Chabot and Francis 2016). The accuracy of scan counts from the ground is likely to decline as colony size increases: distant birds are likely to be obscured behind those closer to the observer and it becomes increasingly difficult for observers to keep track of which nests have been counted (Hodgson et al. 2016). Increasing distances from the observer in larger colonies will also increase the likelihood of identification errors, and nesting birds can more easily be obscured by topography or vegetation (McClelland et al. 2016). Errors can also be inconsistent among species and locations: UAV and ground counts for the two South Georgia Shag colonies at Signy, differed by as little as $<1 \%$ at Shagnasty Island and as much as around $8 \%$ at North Point. We believe this is due to inaccuracies in the ground counts at North Point, where a number of nests on cliff ledges were not visible to the personnel carrying out the ground count. This undercounting by the ground survey at one of the two colonies consequently led to the combined population difference of $\sim 8 \%$ between the two methods. In contrast, in the case of the Gentoo Penguin survey, the close similarity of ground and UAV counts we report was expected, since at Signy this species builds large, clearly defined nests on exposed ground in small colonies ( $<360$ pairs) where there are no features obscuring visibility.

We demonstrate that low altitude multi-rotor UAV surveys are capable of obtaining sufficiently high-resolution images to allow accurate identification of Chinstrap and Adélie Penguins from appearance, despite both species looking very similar from above and the boundaries of several colonies being contiguous. This would not be possible from higher altitude surveys using fixed-wing UAVs or manned aircraft, which would instead depend on other cues such as nest spacing, habitat or guano colouration to separate species (Trathan 2004; Pfeifer et al. 2019). This is more likely to result in identification errors unless the species-specific colony boundaries are first ground-truthed (Waluda et al. 2014).

Aerial images taken by UAVs can provide a permanent record for future reference, facilitating careful and reliable examination of every visible nest: count units can be marked to avoid double and repeat counting, allowing a systematic review of the entire colony and the establishment of clearly defined subdivisions within its boundaries (Hodgson et al. 2018; Rush et al. 2018). The creation of a photographic map also facilitates the study of additional variables such as colony site selection, nest spacing patterns and density (Henriksen et al. 2015; Chabot and Francis 2016).

In our study, manual counting of AONs from images by experienced observers familiar with the species enabled accurate counts to be made from the UAV images, as has been done in previous surveys (Sarda-Palomera et al. 2012; Hodgson et al. 2016, 2018). This was feasible for the relatively small number and sizes of the colonies being surveyed but could become an onerous task in situations where much larger mixed colonies occur. Computer vision is capable of identifying and counting single-species colonies (Trathan 2004; McClelland et al. 2016; Borowicz et al. 2018) and has already been proven to work with gull species of similar appearance in mixed colonies (Rush et al. 2018). However, the extreme similarity between Adélie and Chinstrap Penguins may present a challenge for automated identification of species, but we aim to investigate the feasibility of this in future work.

This study confirms a continuing and significant longterm decline in Chinstrap Penguin breeding pairs at Signy since the late 1970s (Dunn et al. 2016), the population having fallen by $70 \%$ up to 2017/2018. The smaller Gentoo Penguin population has also continued a long-term trend (Dunn et al. 2016), in this case increasing by $\sim 150 \%$ over the same period. The ongoing changes in population trends of these two Pygoscelis penguin populations at Signy, coupled with similar population trends for the same species at other sites across the Scotia Arc and West Antarctic Peninsula (Hinke et al. 2007; Barbosa et al. 2012; Lynch et al. 2012; Lynch 2013; Clucas et al. 2014), highlight the importance of continued and comprehensive long-term monitoring, at Signy and elsewhere, which is essential if we are to understand the large-scale processes that act as drivers of these changes.

Our study provides impetus to extend UAV survey work in the South Orkney region. Additional future UAV surveys, potentially with ground counts for further validation, of the declining Adélie Penguin and Southern Giant Petrel populations at Signy (Dunn et al. 2015, 2016), would be desirable, particularly as the latter is highly sensitive to observers approaching colonies on foot. Previously, the all-island 
surveys of Chinstrap and Adélie Penguins at Signy have been limited to an approximately decadal time frame, owing to the scale of the task of performing these using ground counts (Dunn et al. 2016). The future use of small, portable multi-rotor UAV platforms such as those used in this study will allow more frequent, entire-island population surveys to be carried out at Signy. Multi-rotor UAV surveys could also be extended to obtain breeding success data, collecting information on individual nest survival by carrying out surveys at the peak of incubation and at the end of the brood period when chicks leave their nest to form crèches. Surveys of near-fledged chicks at the end of the crèche period could be used to quantify productivity (chicks fledged per pair) at the sub-colony level.

This study used UAVs to extend surveys beyond the mainland of Signy to include small nearshore islets, that have been inaccessible since cessation of boat operations in the 1990s. Future use of multi-rotor UAVs with greater flight endurance would allow surveys to extend across the Moe Island Antarctic Special Protection Area (ASPA) or nearby colonies on Coronation Island to the north. The portability and speed of deployment of multi-rotor UAVs also allow them to obtain accurate surveys during brief visits to colonies from expedition or tourist ships, extending sampling more widely. Use of fixed-wing UAVs has potential to survey colonies across the whole of the South Orkneys when operating from the bases at Signy and Laurie Islands, and improvements in camera technology may soon allow species identification from the higher altitudes these operate at. The South Orkneys are particularly poorly surveyed for seabirds owing to only two research stations being present in the whole archipelago and visits by tourist ships that provide researchers with access to sites across much of the South Shetlands and Antarctic Peninsula being rare (Naveen et al. 2000; Lynch et al. 2008; Clucas et al. 2014). As a consequence, many sites have not been visited since brief yacht surveys in the 1980s (Poncet and Poncet 1985). UAV surveys are therefore poised to revolutionise surface nesting seabird surveys at the South Orkneys in particular but also more widely across Antarctica.

Supplementary Information The online version contains supplementary material available at https://doi.org/10.1007/s00300-021-02831-6.

\begin{abstract}
Acknowledgements We thank all the staff of the British Antarctic Survey (BAS) Signy Island Research Station who have contributed to and supported the UAV penguin monitoring survey. We also thank Carl Robinson and Peter Enderlein for their technical support, advice and equipment provision and Laura Gerrish for her mapping assistance. This study is part of the Ecosystems component of the British Antarctic Survey Polar Science for Planet Earth Programme, funded by The Natural Environment Research Council.
\end{abstract}

Author contributions MJD, NR and PNT conceived and designed the research. SA, APT and MJD contributed to the fieldwork. MJD, NR and AGW contributed to the data analyses. All authors contributed to and approved the written manuscript.

\section{Compliance with ethical standards}

Ethical approval Our research was approved by the British Antarctic Survey Animal Ethics Review Committee, and permission was granted by the British Foreign and Commonwealth Office on behalf of HM Secretary of State, under Sect. 12 and 13 of the Antarctic Act, 1994, 2013. All relevant data are available within this paper and associated supplementary material. Survey images are available (Dunn et al. 2021) at [https://doi.org/fxbs].

Open Access This article is licensed under a Creative Commons Attribution 4.0 International License, which permits use, sharing, adaptation, distribution and reproduction in any medium or format, as long as you give appropriate credit to the original author(s) and the source, provide a link to the Creative Commons licence, and indicate if changes were made. The images or other third party material in this article are included in the article's Creative Commons licence, unless indicated otherwise in a credit line to the material. If material is not included in the article's Creative Commons licence and your intended use is not permitted by statutory regulation or exceeds the permitted use, you will need to obtain permission directly from the copyright holder. To view a copy of this licence, visit http://creativecommons.org/licenses/by/4.0/.

\section{References}

Barbosa A, Benzal J, De León A, Moreno J (2012) Population decline of chinstrap penguins (Pygoscelis antarctica) on Deception Island. South Shetlands, Antarctica Polar Biol. https://doi.org/10.1007/ s00300-012-1196-1

Barr JR, Green MC, DeMaso SJ, Hardy TB (2018) Detectability and visibility biases associated with using a consumer-grade unmanned aircraft to survey nesting colonial waterbirds. J Field Ornithol. https://doi.org/10.1111/jofo.12258

Black C, Collen B, Lunn D, Filby D, Winnard S, Hart T (2018) Timelapse cameras reveal latitude and season influence breeding phenology durations in penguins. Ecology and Evolution. https://doi. org/10.1002/ece 3.4160

Borowicz A et al (2018) Multi-modal survey of Adelie penguin megacolonies reveals the Danger Islands as a seabird hotspot. Sci Rep 8:3926. https://doi.org/10.1038/s41598-018-22313-w

Borrelle SB, Fletcher AT (2017) Will drones reduce investigator disturbance to surface-nesting birds? Mar Ornithol 45:89-94

Brisson-Curadeau E, Bird D, Burke C, Fifield DA, Pace P, Sherley RB, Elliott KH (2017) Seabird species vary in behavioural response to drone census. Sci Rep. https://doi.org/10.1038/s41598-01718202-3

Buckland ST, Burt ML, Rexstad EA, Mellor M, Williams AE, Woodward R (2012) Aerial surveys of seabirds: the advent of digital methods. J Appl Ecol 49:960-967. https://doi.org/10.111 1/j.1365-2664.2012.02150.x

Callaghan CT, Branis KJ, Lyons MB, Ryall S, Kingsford RT (2018) A comment on the limitations of UAVs in wildlife research - the example of colonial nesting watwerbirds. J Avian Biol. https://doi. org/10.1111/jav.01825

Carney KM, Sydeman WJ (1999) A review of human disturbance effects on nesting colonial waterbirds. Waterbirds 22:68-79

CCAMLR (2004) Standard methods for monitoring parameters of predator species. www.ccamlr.org. Accessed 30 June 2017 
Chabot D, Francis CM (2016) Computer-automated bird detection and counts in high-resolution aerial images: a review. J Field Ornithol 87:343-359. https://doi.org/10.1111/jofo.12171

Chabot D, Shawn RC, Bird DM (2015) Population census of a large common tern colony with a small unmanned aircraft. PLoS ONE. https://doi.org/10.1371/journal.pone.0122588

Clucas GV et al (2014) A reversal of fortunes: climate change 'winners' and 'losers' in Antarctic Peninsula penguins. Sci Rep 4:5024. https://doi.org/10.1038/srep05024

Croxall JP, Butchart SHM, Lascelles B, Stattersfield AJ, Sullivan B, Symes A, Taylor P (2012) Seabird conservation status, threats and priority actions: a global assessment Bird. Conserv Int 22:1-34. https://doi.org/10.1017/S0959270912000020

Ducklow HW et al (2007) Marine pelagic ecosystems: The West Antarctic Peninsula Philos Trans R Soc Lond. B, Biol Sci 362:67-94. https://doi.org/10.1098/rstb.2006.1955

Dunn M (2020) Counts of South Georgia shag Leucocarbo [atriceps] georgianus nests at Signy Island. South Georgia. https://doi. org/10.5285/CA60BFEC-9070-4967-920E-0C42554AD649

Dunn MJ, Jackson JA, Adlard S, Phillips RA (2015) Population size and trends of southern giant petrels (Macronectes giganteus) nesting at Signy Island. South Orkney Islands Polar Biol. https://doi. org/10.1007/s00300-015-1855-0

Dunn MJ, Jackson JA, Adlard S, Lynnes AS, Briggs DR, Fox D, Waluda CM (2016) Population size and decadal trends of three penguin species nesting at Signy Island. South Orkney Islands PLoS ONE 11:e0164025. https://doi.org/10.1371/journ al.pone. 0164025

Dunn MJ, Adlard S, Taylor AP, Wood AG, Trathan PN, Ratcliffe N (2021) Un-crewed Aerial Vehicle (UAV) derived population counts of gentoo and chinstrap penguin and South Georgia shag colonies on Signy Island, South Orkney Islands, 2016-2018. UK Polar Data Centre, Natural Environment Research Council, UK Research \& Innovation, https://doi.org/10.5285/f3d9004a-e7884104-a1a9-54fa80b533a1

Forcada J, Trathan P (2009) Penguin responses to climate change in the Southern Ocean. Global Change Biol 15:1618-1630

Forcada J, Trathan PN, Reid K, Murphy EJ (2006) Contrasting population changes in sympatric penguin species in association with climate warming Global. Change Biol 12:411-423

Fraser WR, Trivelpiece WZ, Ainley DG, Trivelpiece SG (1992) Increases in Antarctic penguin populations - reduced competition with whales or a loss of sea ice due to environmental warming. Polar Biol 11:525-531

Frederick PC, Hylton B, Heath JA, Ruane R (2003) Accuracy and variation in estimates of large numbers of birds by individual observers using an aerial survey simulator. J Field Ornithol 74:281-287

Fretwell PT et al (2012) An Emperor Penguin Population Estimate: The First Global, Synoptic Survey of a Species from Space. PLoS ONE 7:e33751. https://doi.org/10.1371/journal.pone.0033751

Fretwell PT, Phillips RA (2015) Brooke MdL, Fleming AH, McArthur A Using the unique spectral signature of guano to identify unknown seabird colonies. Remote Sens Environ 156:448-456

Fretwell PT, Scofield P, Phillips RA (2017) Using super-high resolution satellite imagery to census threatened albatrosses. Ibis. https://doi. org/10.1111/ibi.12482

Goebel ME, Perryman WL, Hinke JT, Krause DJ, Hann NA, Gardner S, LeRoi DJ (2015) A small unmanned aerial system for estimating abundance and size of Antarctic predators. Polar Biol 38:619-630

Harris CM et al (2015) Important Bird Areas in Antarctica 2015. BirdLife International and Environmental Research \& Assessment Ltd., Cambridge

Henriksen MVJ, Hangstrup S, Work F, Krogsgaard MK, Groom GB, Fox AD (2015) Flock distributions of Lesser Flamingos Phoeniconaias minor as potential responses to food abundance-predation risk trade-offs at Kamfers Dam. South Africa Wildfowl 65:3-18
Hinke JT et al (2018) Estimating nest-level phenology and reproductive success of colonial seabirds using time-lapse cameras. Methods Ecol Evol 9:1853-1863. https://doi. org/10.1111/2041-210X.13015

Hinke JT, Salwicka K, Trivelpiece SG, Watters GM, Trivelpiece WZ (2007) Divergent responses of Pygoscelis penguins reveal a common environmental driver. Oecologia 153:845-855

Hodgson JC, Baylis S, Mott R, Herrod A, Clarke RH (2016) Precision wildlife monitoring using unmanned aerial vehicles. Sci Rep 6:22574. https://doi.org/10.1038/srep22574

Hodgson JC, Koh LP (2016) Best practrice for minimising unmanned aerial vehicle disturbance to wildlife in biological field research. Curr Biol. https://doi.org/10.1016/j.cub.2016.04.001

Hodgson JC et al (2018) Drones count wildlife more accurately and precisely than humans. Mehtods in Ecology and Evolution. https ://doi.org/10.1111/2041-210-x.12974

Junda J, Greene E, Bird D (2015) Proper flight technique for using a small rotary-winged drone aircraft to safely, quickly and accurately survey raptor nests. Journal of Unmanned Vehicle Systems 3:222-236. https://doi.org/10.1139/juvs-2015-0003

Korczak-Abshire M, Zmarz A, Rodzewicz M, Kycko M, Karsznia I, Chwedorzewska KJ (2019) Study of fauna population changes on Penguin Island and Turret Point Oasis (King George Island, Antarctica) using an unmanned aerial vehicle. Polar Biol 42:217-224. https://doi.org/10.1007/s00300-018-2379-1

LaRue MA et al (2014) A method for estimating colony sizes of Adélie penguins using remote sensing imagery. Polar Biol 37:507-517. https://doi.org/10.1007/s00300-014-1451-8

Linchant J, Lisein J, Semeki J, Lejeune P, Vermeulen C (2015) Are unamanned aircraft systems (UASs) the future of wildlife monitoring? Mammal Review, A review of accomplishments and challenges. https://doi.org/10.1111/mam.12046

Lynch HJ (2013) The gentoo penguin (Pygoscelis papua). In: Borboroglu PG, Boersma PD (eds) Penguins: natural history and conservation. University of Washington Press, Seattle, USA, pp 73-88

Lynch HJ, Naveen R, Fagan WF (2008) Censuses of penguin, blue eyed shag Phalacrocorax atriceps and southern giant petrel Macronectes giganteus populations on the Antarctic Peninsula, 2001-2007 Mar Ornithol 36:83-97

Lynch HJ, Naveen R, Trathan PN, Fagan WF (2012) Spatially integrated assessment reveals widespread changes in penguin populations on the Antarctic Peninsula. Ecology 93:1367-1377

Lynch HJ, Ratcliffe N, Passmore J, Foster E, Trathan PN (2013) Sensitivity analysis identifies high influence sites for estimates of penguin krill consumption on the Antarctic Peninsula. Antarct Sci 25:19-23. https://doi.org/10.1017/S095410201200060

Lynch TP, Alderman R, Hobday AJ (2015) A high-resolution panorama camera system for monitoring colony-wide seabird nesting behaviour. Methods Ecol Evol 6:491-499. https://doi. org/10.1111/2041-210x.12339

Magness DR, Eskelin T, Laker M, Renner HM (2019) Evaluation of small unamnned aerial systems as a census tool for Aleutian tern Onychoprion aleuticus colonies. Mar Ornithol 47:11-16

McClelland GTW, Bond AL, Sardana A, Glass T (2016) Rapid population estimate of a surface-nesting seabird on a remote island using a low-cost unamnned aerial vehicle. Mar Ornithol 44:215-220

Mustafa O, Barbosa A, Krause DJ, Peter HU, Vieira G, Rummler MC (2018) State of knowledge: Antarctic wildlife response to unmanned aerial systems. Polar Biol 41:2387-2398. https://doi. org/10.1007/s00300-018-2363-9

Naveen R, Forrest SC, Dagit RG, Blight LK, Trivelpiece WZ, Trivelpiece SG (2000) Censuses of penguin, blue-eyed shag, and southern giant petrel populations in the Antarctic peninsula region 1994-2000. Polar Rec 36:323-334. https://doi.org/10.1017/S0032 247400016818 
Oosthuizen WC, Kruger L, Jouanneau W, Lowther AD (2020) Unmanned aerial vehicle (UAV) survey of the Antarctic shag (Leucocarbo bransfieldensis) breeding colony at Harmony Point. South Shetland Islands Polar Biol 43:187-191

Pfeifer C, Barbosa A, Mustafa O, Peter HU, Rummler MC, Brenning A (2019) Using Fixed-Wing UAV for Detecting and Mapping the Distribution and Abundance of Penguins on the South Shetlands Islands. Antarctica Drones. https://doi.org/10.3390/drones3020 039

Phillips RA et al (2016) The conservation status and priorities for albatrosses and large petrels. Biol Conserv 201:169-183. https://doi. org/10.1016/j.biocon.2016.06.017

Poncet S, Poncet J (1985) A survey of penguin breeding populations at the South Orkney Islands British Antarctic Survey. Bulletin 68:71-81

Ratcliffe N, Guihen D, Robst J, Crofts S, Stanworth A, Enderlein P (2015) A protocol for the aerial survey of penguin colonies using UAVs. Journal of Unmanned Vehicle Systems 3:95-101. https:// doi.org/10.1139/juvs-2015-0006

Rummler MC, Mustafa O, Maercker J, Peter HU, Esefeld J (2015) Measuring the influence of unamanned aerial vehicles on Adelie penguins. Polar Biol. https://doi.org/10.1007/s00300-015-1838-1

Rummler MC, Mustafa O, Maercker J, Peter HU, Esefeld J (2018) Sensitivity of Adelie and gentoo penguins to various flight activities of a micro UAV. Polar Biol 41:2481-2493. https://doi. org/10.1007/s00300-018-2385-3

Rush GP, Clarke LE, Stone M, Wood MJ (2018) Can drones count gulls? Minimal disturbance and semiautomated image processing wih an unmanned aerial vehicle for colony nesting seabirds. Ecology and Evolution 8:12322-12334. https://doi.org/10.1002/ ece 3.4495

Sarda-Palomera F, Bota G, Pallares O, Sazatornil V, Brotons L, Gomariz S, Sarda F (2012) Fine-scale bird monitoring from light unamanned aircraft systems. Ibis 154:177-183

SCAR (2009) Antarctic Climate Change and the Environment (ACCE) Review Report. Available at: https://www.scar.org/policy/acceupdates/

Southwell C, Emmerson LM, Takahashi A, Barbraud C, Delord K, Weimerskirch H (2017a) Large-scale population assessment informs conservation management for seabirds in Antarctica and the Southern Ocean: A case study of Adelie penguins. Global Ecology and Conservation 9:104-115. https://doi.org/10.1016/j. gecco.2016.12.0

Southwell C, Emmerson LM, Takahashi A, Kato A, Barbraud C, Delord K, Weimerskirch H (2017b) Recent studies overestimate colonization and extinction events for AdeliePenguin breeding colonies. Auk 134:39-50. https://doi.org/10.1642/AUK-16-125

Southwell C, McKinlay J, Low M, Wilson D, Newbery K, Lieser JL, Emmerson L (2013) New methods and technologies for regional-scale abundance estimation of land-breeding marine animals: application to Adelie penguin populations in East Antarctica. Polar Biol 36:843-856. https://doi.org/10.1007/s0030 0-013-1310-z

Stammerjohn SE, Martinson DG, Smith RC, Iannuzzi RA (2008a) Sea ice in the Western Antarctic Peninsula region: spatio-temporal variability from ecological and climate change perspectives Deep Sea Res Part II. Top Stud Oceanogr 55:2041-2058

Stammerjohn SE, Martinson DG, Smith RC, Yuan X, Rind D (2008b) Trends in Antarctic annual sea ice reatreat and advance and their relation to El Nino-Southern Oscillation and Southern Annular Mode variability. J Geophys Res-Oceans 113:1-20
Trathan PN (2004) Image analysis of color aerial photography to estimate penguin population size. Wildl Soc Bull 32:332-343

Trathan PN, Croxall JP, Murphy EJ (1996) Dynamics of Antarctic penguin populations in relation to inter-annual variability in sea ice distribution. Polar Biol 16:321-330

Trathan PN, Forcada J, Murphy EJ (2007) Environmental forcing and Southern Ocean marine predator populations: effects of climate change and variability Philos Trans R Soc Lond. B, Biol Sci 362:2351-2365

Trathan PN et al (2015) Pollution, habitat loss, fishing, and climate change as critical threats to penguins. Conserv Biol 29:31-41. https://doi.org/10.1111/cobi.12349

Trathan PN, Murphy EJ, Forcada J, Croxall JP, Reid K, Thorpe SE (2006) Physical forcing in the southwest Atlantic: ecosystem control In: Boyd IL, Wanless S, Camphyusen CJ (eds) Top predators in marine ecosystems: their role in monitoring and management. Cambridge University Press, pp 28-45

Trivelpiece ZW, Hinke JT, Miller AK, Reiss CS, Trivelpiece SG (2011) Variability in krill biomass links harvesting and climate warming to penguin population changes in Antarctica P Natl Acad Sci USA 108:7625-7628

Turner J et al (2016) Absence of 21st century warming on Antarctic Peninsula consistent with natural variability. Nature 535:411-415. https://doi.org/10.1038/nature18645

Valle RG, Scarton F (2020) Drones improve the effectiveness and reduce disturbance of censusing Common Redshanks Tringa totanus breeding on salt marshes. Ardea 107:275-282. https:// doi.org/10.5253/arde.v107i3.a3

Vas E, Lescroel A, Duriez O, Boguszewski G, Gremillet D (2018) Approaching birds with drones: first experiments and ethical guidelines. Biol Let 11:20140754. https://doi.org/10.1098/ rsbl.2014.0754

Vaughan DG et al (2003) Recent rapid regional climate warming on the Antarctic Peninsula. Clim Change 60:243-274

Verfuss UK et al (2019) A review of unmanned vehicles for the detection and monitoring of marine fauna. Mar Pollut Bull. https://doi. org/10.1016/j.marpolbul.2019.01.009

Volkman NJ, Trivelpiece W (1981) Nest site selection among Adelie, chinstrap and gentoo penguins in mixed species rookeries. Wilson Bulletin 93:243-248

Waluda CM, Dunn MJ, Curtis C, Fretwell PT (2014) Assessing penguin colony size and distribution using digital mapping and satellite remote sensing. Polar Biol 37:1849-1855

Weimerskirch H, Prudor A, Schull Q (2018) Flights of drones over subAntarctic seabirds show species-and status-specific behaviourla and physiological responses. Polar Biol 41:259-266. https://doi. org/10.1007/s00300-017-2187-z

White MG, Conroy JWH (1975) Aspects of competition between pygoscelid penguins at Signy Island, Soouth Orkney Islands. Ibis 117:371-373

Williams TD (1995) The Penguins. Oxford University Press, Oxford

Woehler EJ, Croxall JP (1997) The status and trends of Antarctic and Sub-Antarctic seabirds Mar Ornithol 25:43-66

Zmarz A, Rodzewicz M, Dabski M, Karsznia I, Korczak-Abshire M, Chwedorzewska KJ (2018) Application of UAV BVLOS remote sensing data for multi-faceted analysis of Antarctic ecosystem. Remote Sens Environ 217:375-388. https://doi.org/10.1016/j. rse.2018.08.031

Publisher's Note Springer Nature remains neutral with regard to jurisdictional claims in published maps and institutional affiliations. 